\title{
Using the Borg rating of perceived exertion scale to grade the intensity of a functional training program of the affected upper limb after a stroke: a feasibility study
}

This article was published in the following Dove Press journal: Clinical Interventions in Aging

\section{Marie-Hélène Milot Guillaume Léonard Hélène Corriveau Johanne Desrosiers}

University of Sherbrooke, School of Medicine and Health Sciences, School of Rehabilitation, Research Center on Aging, Sherbrooke, Québec, Canada
Correspondence: Marie-Hélène Milot University of Sherbrooke, School of Medicine and Health Sciences, School of Rehabilitation, Research Center on Aging, 1036 Belvédère sud, Sherbrooke, Québec JIH 4C4, Canada

$\mathrm{Tel}+\mathrm{I} 8197802220$

Fax $+|8| 98297|4|$

Email marie-helene.milot@ usherbrooke.ca
Purpose: Intensity of a training program is a critical variable in treatment gains poststroke, but there are no guidelines to adequately dose the intensity of functional training (FT); the recommended type of training to promote poststroke recovery. Such guidelines are made available for strength training (ST) using the 1 repetition maximum (1RM), which has been linked to individuals' self-rated level of exertion using the Borg rating of perceived exertion (BRPE) scale. The BRPE could be a valuable tool for clinicians to dose FT intensity after a stroke, but this remains to be tested. The main objective of the study was to evaluate the feasibility of the BRPE at grading FT intensity of the affected upper limb in older adults with a chronic stroke and secondarily to explore the clinical changes between FT and ST when the intensity is regulated with BRPE.

Patients and methods: Twelve participants were randomized into a FT or ST group and trained their affected upper limb (3 times/week for 4 weeks) with the intensity standardized with BRPE. Feasibility was assessed by adherence, occurrence of adverse events, and comparison of BRPE ratings between groups. Clinical changes were defined as improvements on the FuglMeyer motor assessment (FMA) and Wolf motor function test (WMFT).

Results: All participants adhered to FT/ST without adverse effects, and comparable BRPE ratings were noted between groups throughout the training $(P \geq 0.42)$. Both groups showed significant gains at the FMA (ST: $5 \pm 4$ points/FT: $6 \pm 4$ points; $P=0.04$ ) and WMFT (ST: $0.4 \pm 0.3$ points/FT: $0.6 \pm 0.4$ points; $P=0.05)$, which were comparable between groups $(P \geq 0.47)$.

Conclusion: The results suggest that it is feasible to use the BRPE scale to adjust FT intensity. Gains in motor function in both groups suggest that undergoing therapy, regardless of its type, might be a sufficient stimulus to produce gains when intensity is adequately adjusted. Further studies are needed to validate the current observations.

Keywords: stroke, Borg rating of perceived exertion scale, functional training, strength training, feasibility, motor function

\section{Introduction}

Age is among the strongest risk factors for stroke. Starting at 55 years, the risk of stroke doubles each decade; hence, about $80 \%$ of strokes happen in individuals aged 60 years and older. ${ }^{1,2}$ Muscle weakness contralateral to the brain lesion is among the most debilitating impairments after stroke and has a detrimental impact on the functional performance and independence of survivors. ${ }^{3}$ North American and European stroke best practice guidelines ${ }^{4,5}$ recommend the prescription of exercise to help reduce the negative impact of muscle weakness on stroke recovery, regardless of age. Knowing that 
the level of participation in an exercise program is one of the best predictors of performance in activities of daily living in older adults, ${ }^{6}$ promoting the practice of exercise becomes even more important for that subset of the population. Exercise can be defined as the practice of movements through a gradual increase in resistance using free weights or machines (strength training [ST]) or by the repetitive practice of a functional task (functional training [FT]). ${ }^{3,4}$ To optimize treatment gains and adherence, the current trend in poststroke rehabilitation is to provide training that is meaningful to the individual. ${ }^{7}$ Today, therefore, FT is the most recommended type of training to support recovery, ${ }^{7,8}$ even at the chronic stage of a stroke. ${ }^{9-12}$

The characteristics (eg, frequency, intensity) of exercise programs are of crucial importance to optimize training responses and promote recovery poststroke. ${ }^{3}$ Among these characteristics, intensity of training is now recognized as a key element of exercise prescription. ${ }^{13}$ So far, intensity has been evaluated mainly by the amount of time spent in the therapy, with augmented therapy time usually translating into further improvement in motor recovery. ${ }^{13}$ Because of the varying degrees of motor recovery that occur after a stroke, similar time spent in the therapy does not necessarily mean similar intensity of training; for the same amount of therapy time, a stroke survivor with better recovery is more likely to do more exercise repetitions than a survivor with a lower level of recovery. ${ }^{13}$ Knowing that FT is the most frequent type of exercise adopted by clinicians during their clients' rehabilitation, it becomes crucial to ensure a proper intensity level for this training program to adequately support recovery. Unfortunately, intensity grading of a FT program can be challenging for clinicians, as no specific guidelines exist so far, ${ }^{7}$ often translating into a suboptimal FT intensity level. ${ }^{14}$ On the other hand, stroke best practice guidelines do provide explicit intensity grading for ST programs, using the 1 repetition maximum (1RM; maximal load that a person can lift once in his available range of motion). ${ }^{8}$ The guidelines state that intensity of ST should start at $50 \%$ of $1 \mathrm{RM}$ and increase to up to $80 \%$ of $1 \mathrm{RM}$ during training. Thus, ST program intensity can be easily controlled to provide challenging exercises at all times during the training of patients with a stroke.

One way to help clinicians set and grade the intensity of their FT programs could be by the use of the Borg rating of perceived exertion (BRPE) scale. ${ }^{15}$ The BRPE is a user-friendly scale that measures an individual's self-rated physical exertion during exercises with scores ranging from 6 (no exertion) to 20 (maximum exertion). ${ }^{15}$ The BRPE can be accurately rated by individuals with a stroke, regardless of the severity of their motor impairments. ${ }^{16-18}$ The BRPE has been positively used to prescribe ST intensity of the $\operatorname{leg}^{19}$ and $\operatorname{arm}^{20}$ in young and older healthy individuals with BRPE scores of 11,13 , and 16/20, representing an intensity level of about $40 \%, 60 \%$, and $80 \%$ of $1 \mathrm{RM}$, respectively. ${ }^{19,20}$ Because the BRPE is a self-rated exertion scale, it considers the individual's residual ability and could represent a preferable way to set and grade the intensity of training poststroke than the time spent in therapy.

So far, the BRPE has never been used to grade the training intensity of a FT program in either healthy people or individuals with a pathology, such as a chronic stroke, to warrant optimal FT training characteristics. Considering the abovementioned studies, we hypothesized that the BRPE would be a feasible scale to use to grade the intensity level of a FT program, while following ST intensity grading recommendations. Also, based on the premise that type of training does not seem to be a key element in promoting posttraining clinical gains after a stroke, ${ }^{3,9,21,22}$ the secondary objective was to explore the effectiveness of FT and ST in improving affected upper limb recovery, when the intensity of training is comparable. We hypothesized that both FT and ST would produce similar improvements in affected upper limb recovery.

\section{Patients and methods Design and participants}

This study took part at the Research Center on Aging in Sherbrooke (Quebec, Canada), and the participants were recruited from the medical archives of the CIUSSS de l'Estrie-CHUS, as well as from the Association des accidentés cérébro-vasculaires et traumatisés crâniens de l'Estrie, an association for people who have suffered a stroke in Sherbrooke. To be part of this single-blind pilot study, participants had to meet the following eligibility criteria: 1) age $\geq 50$ years, 2 ) single stroke $\geq 6$ months prior to study entry, 3) present at least minimal motor recovery in the affected upper limb ( $\geq 15 / 66$ on the Fugl-Meyer motor assessment [FMA]), and 4) have completed any rehabilitation treatment. People with 1) contracture of the affected upper limb (score $>3$ on the modified Ashworth Scale at the elbow, wrist, fingers, and thumb flexors), 2) orthopedic problems in the affected upper limb (eg, fracture, sprain), and 3) neurological problems other than the stroke were excluded. All participants signed a written informed consent form, and the study was approved by the CIUSSS de l'EstrieCHUS institutional ethics board. This study was conducted in accordance with the Declaration of Helsinki. 
Participants were stratified based on their baseline FMA score $(<40$ and $\geq 40$ ). Within each stratum, a computerized simple randomization was then performed by one of the authors (M-H M) to assign participants to the ST group (n=6) or the FT group $(n=6)$. Group allocation was concealed by the author in a spreadsheet.

\section{Training programs and feasibility}

The training parameters were identical for both training programs and followed the American Stroke Association position on exercise prescription after stroke. ${ }^{8}$ Participants were trained three times a week for four weeks. Each training session comprised three series of 10 repetitions for each movement, with a 2-minute rest between series. Each training session lasted 60 minutes. For both training groups, muscle groups that play a key role in the functional performance of the upper limb were targeted ${ }^{23,24}$ (ie, wrist extensors, elbow and shoulder flexors, and grip muscles).

The intensity of training was standardized using the BRPE 6-20 scale. ${ }^{18}$ The BRPE 6-20 scale was preferred over the BRPE 0-10 scale because previous studies used the same scale for evaluating the link between perceived exertion and 1RM. ${ }^{19,20}$ After each set of exercises, the trainer asked the participant to rate his level of effort at performing the exercises on the BRPE scale ("How hard you feel your body has worked?"). Shortness of breath was not a variable to consider in the rating, as done so for aerobic exercise. ${ }^{25}$ To follow the ST intensity training guidelines, the intensity level of the FT program started at a BRPE of 12-13/20 ("fairly light/"somewhat hard", respectively) to reach 15-16/20 ("hard"/“"very hard", respectively) by week 4, corresponding to about $80 \%$ of 1 RM. ${ }^{19,20}$

Feasibility of the study was assessed by noting participants' adherence to the training program, when using the BRPE scale to grade the intensity of the FT program, by comparing the participants' BRPE ratings in FT and ST groups after each week of training and by recording the occurrence of adverse events (eg, pain, spasticity).

\section{Strength training program}

For the ST, participants trained the shoulder flexors, elbow flexors, and wrist extensors by lifting free weights with the targeted muscle groups. Grip muscles were trained using a JAMAR ${ }^{\circledR}$ dynamometer (Lafayette, IN, USA). For each muscle group, the maximum load that a participant could lift 10 times (10RM) was used to estimate the 1RM using the formula of Brzycki. ${ }^{26}$ The 10RM was preferred over the $1 \mathrm{RM}$ to avoid tendon and muscle injuries as well as fatigue, knowing that hemiparetic muscles undergo structural and metabolic changes due to the stroke. ${ }^{27}$ At the beginning of each week of training, the 1RM was estimated de novo to allow proper dosing and progression of the free weights lifted during shoulder flexors, elbow flexors, and wrist extensors training. For the grip muscles, participants' maximum grip strength was determined each week and used to dose and determine the progression of the training of the grip muscles. Training started at $50 \%$ of subjects $1 \mathrm{RM}$ or maximal grip strength and progressed to $60 \%, 70 \%$ and $80 \%$ at weeks 2 , 3 and 4 , respectively.

\section{Functional training program}

The FT aimed at performing various tasks involving the affected upper limb such as writing, pouring water, screwing, lifting boxes and putting them on a shelf, and throwing and catching a Velcro ball. Progression was made when the participant rating was below the BRPE target intensity level for each specific week of training. For example, for week 1, progression of exercises was made if the subject rated a set of tasks $\leq 12$ on the BRPE. The trainer then increased the difficulty of the task to keep it challenging, but feasible for the participant by varying, among other things, the speed of movement or size of an object. For each participant, the trainer recorded training progression in a log book. Training started at a BRPE rating of $12-13 / 20$ and progressed to $13-14 / 20$, $14-15 / 20$, and $15-16 / 20$ at weeks 2,3 , and 4 , respectively.

\section{Clinical assessment}

Before and after FT and ST, participants underwent a clinical assessment of their affected upper limb by a blinded evaluator. The main outcome measures were the scores in the FMA, assessing motor function of the affected upper limb $(66=$ normal $),{ }^{28}$ and the Wolf motor function test (WMFT), ${ }^{29}$ evaluating functional performance of the affected upper limb through 15 functional tasks ( $5=$ normal) and 2 strength-based tasks. These tools have good validity and reliability with people with a stroke..$^{30-32}$

The secondary outcome measures were the scores in the Motor Activity Log (MAL), ${ }^{33}$ indicating the participants' self-rated amount and quality of use of their affected upper limb in daily activities ( $5=$ normal), and grip strength, measured with a JAMAR ${ }^{\circledR}$ dynamometer (average of three trials in kilograms).

\section{Statistical analyses}

Feasibility outcome measures were assessed by summarizing the number of participants having completed the FT 
and ST, as well as the number of adverse events reported. Also, for each week of training, each subject's BRPE ratings within a training session were averaged, and the mean (SD) of the BRPE ratings per week of training for each group was calculated. Comparison of these ratings between both groups was done by a Wilcoxon rank-sum test. To explore the impact of FT and ST on clinical measures, Mann-Whitney $U$ and Chi-squared tests were first used to ensure that both groups were comparable on sociodemographic and clinical outcomes. Afterward within each group, clinical changes were evaluated by a Wilcoxon signed-rank test, whereas a Wilcoxon rank-sum test was used to compare these clinical changes between the two groups. Significance level was set at 0.05 , and statistical analyses were performed using SPSS $18^{\circledR}$ (SPSS Inc., Chicago, IL, USA).

\section{Results}

\section{Feasibility}

All participants fully complied with the FT and ST training programs and completed all 12 training sessions (100\% adherence). Moreover, no participant reported the occurrence of adverse events in the FT and ST training groups, and no exacerbation of spasticity in the affected upper limb was noted for either group posttraining. For each week of training, the FT group BRPE ratings were similar to the ST group (Table 1). The FT group average BRPE rating reached $13 \pm 1.4$ at week 1 and $14 \pm 1.6$ at week 4 , whereas the ST group BRPE rating reached $13 \pm 0.6$ at week 1 and $14 \pm 1.2$ at week 4 (Table 1).

\section{Clinical changes}

\section{Baseline sociodemographic and clinical characteristics}

All the subjects presented sufficient motor recovery of the affected upper limb (FMA scores range from 17 to 66/66)

Table I BRPE ratings of the strength and functional training groups

\begin{tabular}{|c|c|c|c|}
\hline $\begin{array}{l}\text { BRPE } \\
\text { rating } \\
(/ 20)\end{array}$ & $\begin{array}{l}\text { Strength training } \\
\text { group, mean } \\
\text { (SD) [range] }\end{array}$ & $\begin{array}{l}\text { Functional training } \\
\text { group, mean (SD) } \\
\text { [range] }\end{array}$ & $P$-value \\
\hline Week I & $\begin{array}{l}13(0.6) \\
{[13-14]}\end{array}$ & $\begin{array}{l}13(1.4) \\
{[10-14]}\end{array}$ & 1.0 \\
\hline Week 2 & $\begin{array}{l}13(0.8) \\
{[12-14]}\end{array}$ & $\begin{array}{l}14(1.5) \\
{[12-16]}\end{array}$ & 1.0 \\
\hline Week 3 & $\begin{array}{l}13(1.0) \\
{[12-14]}\end{array}$ & $\begin{array}{l}14(1.5) \\
{[12-16]}\end{array}$ & 1.0 \\
\hline Week 4 & $\begin{array}{l}14(1.2) \\
{[12-16]}\end{array}$ & $\begin{array}{l}14(1.6) \\
{[11-15]}\end{array}$ & 1.0 \\
\hline
\end{tabular}

Abbreviation: BRPE, Borg rating of perceived exertion. to exercise the targeted muscle groups. No statistically significant difference was noted between the two groups for baseline sociodemographic and clinical characteristics, except for spasticity of the affected wrist flexors, where the FT group showed more spasticity than the ST group. However, the mean score on the modified Ashworth Scale for the wrist flexors was very low, falling below the category of "slight increase in muscle tone"; even though the FT group presented more spasticity, the wrist joint could still be moved easily in both groups (Table 2).

\section{Functional training group}

The FT group presented a significant 6-point gain on the FMA, exceeding the minimal clinically important difference (MCID) of five points for this scale at the chronic phase of a stroke. ${ }^{34}$ Only the score, and not the time to completion, showed improvement on the WMFT, falling beyond the MCID of 0.3 points for individuals with a chronic stroke. ${ }^{35}$ This means that, in general, participants performed better at the tasks but did so at the same speed. For the MAL, although participants reported no change in the amount of use of their affected upper limb in daily tasks posttraining, they noted a significant improvement in the quality of its use. No change in the affected grip strength was observed posttraining (Table 3).

\section{Strength training group}

Similar to the FT group, the ST group showed a significant 5-point gain on the FMA, reaching the FMA MCID cutoff score. ${ }^{34}$ Significant improvements in the WMFT score, time to completion, and amount of weight that could be lifted were also noted, exceeding the 0.3 points and 1.5 seconds WMFT MCID cutoff scores. ${ }^{35}$ These changes were accompanied by a significant improvement in quality of use on the MAL of the affected upper limb, as self-reported by the participants, with a trend toward a positive change for the amount of use. Of note, an average score greater than three points on the MAL can be indicative of a meaningful improvement posttherapy, where the individual can perform 50\% more tasks with his affected upper limb without assistance. ${ }^{36,37}$ No change was noted in affected grip strength (Table 3 ).

\section{Group comparison}

No difference in clinical changes was noted between the two groups, except for the weight that could be lifted with the affected upper limb on the WMFT, which increased significantly in the ST group (Table 3). 
Table 2 Baseline sociodemographic and clinical characteristics of the strength and functional training groups

\begin{tabular}{|c|c|c|c|}
\hline & Strength training group & Functional training group & $P$-value \\
\hline \multicolumn{4}{|l|}{ Sociodemographic characteristics } \\
\hline Age (years) & $64 \pm 9$ & $68 \pm 6$ & 0.52 \\
\hline Time since stroke (months) & $47 \pm 18$ & $58 \pm 79$ & 0.52 \\
\hline Side of stroke (right/left) & $1 / 5$ & $2 / 4$ & $0.08^{\mathrm{a}}$ \\
\hline \multicolumn{4}{|l|}{ Clinical characteristics } \\
\hline Fugl-Meyer motor assessment (normal=66) & $50 \pm 17$ & $56 \pm 7$ & 0.63 \\
\hline \multicolumn{4}{|l|}{ Wolf motor function test } \\
\hline Score $($ normal=5) & $3.7 \pm 1.3$ & $3.9 \pm 0.6$ & 1.0 \\
\hline Time to completion (s) & $13.6 \pm 25.6$ & $3.1 \pm 0.9$ & 0.75 \\
\hline Weight (maximum=20 lbs) & $9 \pm 6$ & $14 \pm 7$ & 0.29 \\
\hline \multicolumn{4}{|l|}{ Motor Activity Log } \\
\hline Amount of use (normal=5) & $3.0 \pm 1.6$ & $3.8 \pm 0.9$ & 0.42 \\
\hline Quality of use (normal=5) & $2.8 \pm 1.5$ & $3.5 \pm 1.0$ & 0.69 \\
\hline \multicolumn{4}{|l|}{ Modified Ashworth Scale } \\
\hline Elbow flexors (normal=0) & $0.7 \pm 0.5$ & $0.8 \pm 0.8$ & 0.72 \\
\hline Wrist flexors (normal=0) & $0 \pm 0$ & $0.8 \pm 0.8$ & 0.02 \\
\hline Fingers flexors (normal=0) & $0.5 \pm 0.8$ & $0.3 \pm 0.8$ & 0.59 \\
\hline Thumb flexors (normal=0) & $0.2 \pm 0.4$ & $0.2 \pm 0.4$ & 1.0 \\
\hline \multicolumn{4}{|l|}{ Grip strength $(\mathrm{kg})$} \\
\hline Affected side & $15 \pm 8$ & $24 \pm 9$ & 0.08 \\
\hline Unaffected side & $28 \pm 12$ & $34 \pm 10$ & 0.42 \\
\hline
\end{tabular}

Note: a Chi-squared test, otherwise Mann-Whitney $U$ test.

\section{Discussion}

This pilot study examined the feasibility of using the BRPE scale to dose the intensity level of a FT program of the affected upper limb in older adults with a chronic stroke.
The BRPE was positively used by all participants, and the FT group ratings were similar to the ones of the ST group that was trained at recommended intensity levels as set by the American Stroke Association 1RM guidelines. Moreover,

Table 3 Changes in clinical measures within and between the strength and functional training groups following a 4-week training program

\begin{tabular}{|c|c|c|c|c|c|c|c|}
\hline \multirow[t]{2}{*}{ Clinical measures } & \multicolumn{2}{|c|}{ Strength training group } & \multirow[t]{2}{*}{$P$-value ${ }^{a}$} & \multicolumn{2}{|c|}{ Functional training group } & \multirow[t]{2}{*}{$P$-value ${ }^{a}$} & \multirow[t]{2}{*}{$P$-value ${ }^{b}$} \\
\hline & Pre & Post & & Pre & Post & & \\
\hline FMA (normal=66) & $50 \pm 17$ & $55 \pm 15$ & 0.04 & $56 \pm 7$ & $62 \pm 5$ & 0.04 & 0.63 \\
\hline \multicolumn{8}{|l|}{ WMFT } \\
\hline Score (normal=5) & $3.7 \pm 1.3$ & $4.1 \pm 1.2$ & 0.05 & $3.9 \pm 0.6$ & $4.5 \pm 0.4$ & 0.05 & 0.47 \\
\hline Time to completion (s) & $13.6 \pm 25.6$ & $10.4 \pm 18.9$ & 0.05 & $3.1 \pm 0.9$ & $2.6 \pm 0.9$ & 0.17 & 0.75 \\
\hline Weight (maximum $=20 \mathrm{lbs}$ ) & $9 \pm 6$ & $13 \pm 7$ & 0.03 & $14 \pm 7$ & $14 \pm 7$ & 0.66 & 0.01 \\
\hline \multicolumn{8}{|l|}{ MAL } \\
\hline Amount of use (normal=5) & $3.0 \pm 1.6$ & $3.6 \pm 1.6$ & 0.08 & $3.8 \pm 0.9$ & $4.2 \pm 0.9$ & 0.14 & 0.75 \\
\hline Quality of use (normal=5) & $2.8 \pm 1.5$ & $3.4 \pm 1.6$ & 0.03 & $3.5 \pm 1.0$ & $4.1 \pm 0.8$ & 0.04 & 0.87 \\
\hline \multicolumn{8}{|l|}{ MAS } \\
\hline Elbow flexors (normal=0) & $0.7 \pm 0.5$ & $0.7 \pm 0.5$ & 1.0 & $0.8 \pm 0.8$ & $0.5 \pm 0.5$ & 0.16 & 0.39 \\
\hline Wrist flexors (normal=0) & $0 \pm 0$ & $0.3 \pm 0.5$ & 0.16 & $0.8 \pm 0.8$ & $0.3 \pm 0.5$ & 0.18 & 0.13 \\
\hline Fingers flexors (normal $=0$ ) & $0.5 \pm 0.8$ & $0.3 \pm 0.8$ & 0.32 & $0.3 \pm 0.8$ & $0.2 \pm 0.4$ & 0.32 & 1.0 \\
\hline Thumb flexors (normal=0) & $0.2 \pm 0.4$ & $0 \pm 0$ & 0.32 & $0.2 \pm 0.4$ & $0.2 \pm 0.4$ & 0.32 & 1.0 \\
\hline \multicolumn{8}{|l|}{ Grip strength (kg) } \\
\hline Affected side & $15 \pm 8$ & $17 \pm 8$ & 0.34 & $24 \pm 9$ & $25 \pm 11$ & 1.0 & 0.57 \\
\hline
\end{tabular}

Notes: aWithin-group comparison using the Wilcoxon signed-rank test. 'Between-group comparison using the Wilcoxon rank-sum test.

Abbreviations: FMA, Fugl-Meyer motor assessment; WMFT, Wolf motor function test; MAL, Motor Activity Log; MAS, Modified Ashworth Scale. 
no adverse events were reported by the participants during training. The secondary objective of this pilot study was to explore the impact of FT and ST on clinical outcomes of the affected upper limb when both groups were trained at a comparable level of intensity. With the exception of grip strength, results showed significant improvements on the FMA, WMFT, and MAL for both groups.

Since intensity in the present study was graded using the BRPE and standardized between FT and ST and that both training groups showed significant clinical gains, this suggests that intensity is an important variable to control in training protocols to ensure gains after a stroke. Unfortunately, recommendations for the dosage of FT program intensity do not exist in current guidelines for exercise prescription after a stroke, ${ }^{8}$ even though FT is the most frequently adopted form of therapy in rehabilitation settings. Adjustment of FT is thus based on clinicians' judgment or time spent in therapy. In their review on dose-response relationship in stroke rehabilitation, Lohse et $\mathrm{al}^{13}$ noted that the time spent in therapy, used as an indicator of treatment intensity, was a significant predictor of improvement after a stroke. However, as reported by Wallace et al, ${ }^{18}$ time spent in therapy can be highly influenced by the survivor's motor recovery and thus might not equate to a similar intensity of treatment between individuals. Hence, in poststroke training protocols, other measures of intensity should be used rather than time spent in therapy, one of the most commonly used measures of intensity reported in the literature to date. ${ }^{13}$ In the present feasibility study, we proposed the use of the BRPE to dose intensity of training for FT programs with a focus on the level of effort of participants during exercises rather than shortness of breath, as used for aerobic exercising. ${ }^{25}$ The BRPE is a user-friendly and low-cost scale that can be adequately rated by individuals with a stroke, ${ }^{16-18}$ as observed in the current study. The overall average rating of participants in the FT group was 14 with a range of $12-15$, falling in the target intensity training of $50 \%-80 \% 1 \mathrm{RM}^{19}$ of ST stroke exercises recommendations. ${ }^{8}$ Since the BRPE was positively used to dose and standardize the intensity of therapy without any adverse events, it could thus be an interesting tool to adjust intensity between training types and individuals.

The finding that FT did not generate greater clinical gains than ST supports previous studies comparing these two types of training. ${ }^{9,21,22}$ It is thought that because of the stroke, individuals are often less active ${ }^{9}$ and deconditioned, ${ }^{38}$ with cardiorespiratory fitness as low as half that of age-matched healthy individuals. Moreover, older adults often have other chronic diseases, impacting their level of physical activity even more. ${ }^{1,38}$ Thus, participating in a demanding training program exercising muscle groups that play a key role in performing daily tasks could be a sufficient stimulus to foster clinical gains of the trained limb, regardless of the training type, ${ }^{9}$ when the intensity of training is properly dosed. Since the present preliminary results seem to show that FT and ST are equally effective, clinicians could then choose a training program that best fits their patients' preference and use the BRPE to set, monitor, and dose the intensity of training to allow their clients to recover to their full potential.

\section{Study limitations}

In general, the participants in this pilot study had recovered well from their stroke. It would be interesting to examine the usability of the BRPE at dosing intensity of training in more severely affected older adults, as well as with individuals with cognitive impairments, to extend the results of the present study to a broader population with a stroke. Of note, since the training program progressed from a BRPE rating of $12-13$ to $15-16 / 20$, subjects did not experience a wide variety of perceived exertion to fully assess the BRPE scale at dosing the intensity of training. In addition, knowing the link between heart rate and perceived exertion during aerobic exercises, ${ }^{15}$ it would have been interesting to evaluate if this relationship still holds for a FT or ST program. However, heart rate was not monitored in this study. Also, clinicians should keep in mind that individuals with a stroke, especially when in the chronic phase, could overestimate their BRPE rating, since their level of activity tends to decrease after a stroke, limiting their conception of maximal exertion. ${ }^{39}$ Thus, clinicians should be careful when using a self-rated physical exertion scale, although no adverse events were reported in the current study, supporting the use of the BRPE at dosing intensity of training. However, because this was a pilot study with a limited number of participants, larger randomized studies are needed to validate the current preliminary observations. Finally, the BRPE might not be optimal for all types of training or activities, since a poor relationship between the BRPE and total energy expenditure, as assessed by gas exchange analysis, was noted for walking and stair activities in individuals with a chronic stroke. ${ }^{39}$

\section{Conclusion}

This pilot study showed that it is feasible to use the BRPE scale as a tool to guide the intensity regulation of a FT program of the upper limb in older adults at the chronic phase of a stroke. When trained at a proper intensity level, both FT and ST translated into gains on clinical measures. 
The results suggest that the type of training might not be a critical feature of training protocols at the chronic stage of a stroke and that training at an appropriate intensity might be a strong enough stimulus to warrant treatment gains. Based on the current feasibility results, a randomized controlled trial, using the present BRPE protocol, is needed to further assess if a FT program, regulated with the BRPE, is more effective at improving recovery after a stroke than a FT program, as currently used in rehabilitation settings. This will allow to provide clinicians with a user-friendly tool to properly train their clients and help them recover to their full potential.

\section{Acknowledgments}

This study was supported by a grant from the Research Center on Aging, Sherbrooke, Quebec, Canada. The research was conducted at the Laboratoire d'évaluation cérébrale et de la douleur (Brain and pain evaluation laboratory) at the Centre de recherche sur le vieillissement (Research Center on Aging), Sherbrooke, Quebec, Canada. The authors offer their heartfelt thanks to the clinicians at the CIUSSS de l'Estrie-CHUS and the Association des accidentés cérébrovasculaires et traumatisés crâniens de l'Estrie, as well as the research staff (Marie-Claude Girard, Anne-Christine Cloutier, Alexandra Gagné-Lauzon, Valérie Laflamme, Roxanne Lapointe-Tremblay, Valérie Lavoie-Giguère, Kayla Vézina-Bergeron, and Amy Bouchard) for their participation in the project. The authors would also like to thank Antoine Guillerand and Mathieu Hamel for their technical support.

\section{Disclosure}

The authors report no conflicts of interest in this work.

\section{References}

1. Heart and Stroke Foundation of Canada. Stroke report. 2017:16. Available from: https://www.heartandstroke.ca/-/media/pdf-files/canada/ stroke-report/strokereport2017en.ashx?1a=en\&hash $=67 \mathrm{~F} 86 \mathrm{E} 4 \mathrm{C} 3338 \mathrm{D}$ 5A7FE7862EA5D0DD57CA8539847. Accessed December 4, 2018.

2. American Stroke Association [homepage on the Internet]. Stroke Risks; 2018. Available from: http://www.strokeassociation.org/STROKEORG/AboutStroke/UnderstandingRisk/Understanding-Stroke-Risk_ UCM_308539_SubHomePage.jsp. Accessed November 21, 2018.

3. Patten C, Lexell J, Brown HE. Weakness and strength training in persons with poststroke hemiplegia: rationale, method, and efficacy. J Rehabil Res Dev. 2004;41(3A):293-312.

4. National Institute for Health and Care Excellence (NICE). Stroke Rehabilitation: clinical guideline. National Clinical Guideline Centre. 2013;591. Available from: https://www.nice.org.uk/guidance/cg162. Accessed December 4, 2018.

5. Hebert D, Lindsay MP, Mcintyre A, et al. Canadian stroke best practice recommendations: Stroke rehabilitation practice guidelines, update 2015. Int J Stroke. 2016;11(4):459-484.

6. Vermeulen J, Neyens JC, van Rossum E, Spreeuwenberg MD, de Witte LP. Predicting ADL disability in community-dwelling elderly people using physical frailty indicators: a systematic review. BMC Geriatr. 2011;11:33
7. Hubbard IJ, Parsons MW, Neilson C, Carey LM. Task-specific training: evidence for and translation to clinical practice. Occup Ther Int. 2009; 16(3-4):175-189.

8. Billinger SA, Arena R, Bernhardt J, et al. Physical activity and exercise recommendations for stroke survivors: a statement for healthcare professionals from the American Heart Association/American Stroke Association. Stroke. 2014;45(8):2532-2553.

9. Milot M-H, Nadeau S, Gravel D, Bourbonnais D. Gait performance and lower-limb muscle strength improved in both upper-limb and lowerlimb isokinetic training programs in individuals with chronic stroke. ISRN Rehabil. 2013;2013(1):1-10.

10. Milot MH, Spencer SJ, Chan V, et al. A crossover pilot study evaluating the functional outcomes of two different types of robotic movement training in chronic stroke survivors using the arm exoskeleton BONES. J Neuroeng Rehabil. 2013;10(1):112.

11. da Silva PB, Antunes FN, Graef P, Cechetti F, Pagnussat AS. Strength training associated with task-oriented training to enhance upperlimb motor function in elderly patients with mild impairment after stroke: a randomized controlled trial. Am J Phys Med Rehabil. 2015; 94(1):11-19.

12. Chang JJ, Tung WL, Wu WL, Huang MH, Su FC. Effects of robotaided bilateral force-induced isokinetic arm training combined with conventional rehabilitation on arm motor function in patients with chronic stroke. Arch Phys Med Rehabil. 2007;88(10):1332-1338.

13. Lohse KR, Lang CE, Boyd LA. Is more better? Using metadata to explore dose-response relationships in stroke rehabilitation. Stroke. 2014;45(7):2053-2058.

14. Dobkin BH. Training and exercise to drive poststroke recovery. Nat Clin Pract Neurol. 2008;4(2):76-85.

15. Borg GA. Psychophysical bases of perceived exertion. Med Sci Sports Exerc. 1982;14(5):377-381.

16. Hampton S, Armstrong G, Ayyar MS, Li S. Quantification of perceived exertion during isometric force production with the Borg scale in healthy individuals and patients with chronic stroke. Top Stroke Rehabil. 2014; 21(1):33-39.

17. Sage M, Middleton LE, Tang A, Sibley KM, Brooks D, Mcilroy W. Validity of rating of perceived exertion ranges in individuals in the subacute stage of stroke recovery. Top Stroke Rehabil. 2013;20(6): 519-527.

18. Wallace AC, Talelli P, Dileone M, et al. Standardizing the intensity of upper limb treatment in rehabilitation medicine. Clin Rehabil. 2010; 24(5):471-478.

19. Lagally KM, Amorose AJ. The validity of using prior ratings of perceive exertion to regulate resistance exercise intensity. Percept Mot Skills. 2007;104(2):534-542.

20. Row Lazzarini BS, Dropp MW, Lloyd W. Upper-extremity explosive resistance training with older adults can be regulated using the rating of perceived exertion. J Strength Cond Res. 2017;31(3): 831-836.

21. Friedman N, Chan V, Reinkensmeyer AN, et al. Retraining and assessing hand movement after stroke using the MusicGlove: comparison with conventional hand therapy and isometric grip training. J Neuroeng Rehabil. 2014;11(1):76.

22. Winstein CJ, Rose DK, Tan SM, Lewthwaite R, Chui HC, Azen SP. A randomized controlled comparison of upper-extremity rehabilitation strategies in acute stroke: a pilot study of immediate and long-term outcomes. Arch Phys Med Rehabil. 2004;85(4):620-628.

23. Boissy P, Bourbonnais D, Carlotti MM, Gravel D, Arsenault BA. Maximal grip force in chronic stroke subjects and its relationship to global upper extremity function. Clin Rehabil. 1999;13(4):354-362.

24. Mercier C, Bourbonnais D. Relative shoulder flexor and handgrip strength is related to upper limb function after stroke. Clin Rehabil. 2004;18(2):215-221.

25. Scherr J, Wolfarth B, Christle JW, Pressler A, Wagenpfeil S, Halle M. Associations between Borg's rating of perceived exertion and physiological measures of exercise intensity. Eur J Appl Physiol. 2013;113(1): $147-155$. 
26. Brzycki M. Strength testing - predicting a one-rep max from reps-tofatigue. J Phys Educ Recreat Dance. 1993;64(1):88-90.

27. Scherbakov N, von Haehling S, Anker SD, Dirnagl U, Doehner W. Stroke induced sarcopenia: muscle wasting and disability after stroke. Int J Cardiol. 2013;170(2):89-94.

28. Fugl-Meyer AR, Jääskö L, Leyman I, Olsson S, Steglind S. The post-stroke hemiplegic patient. 1. a method for evaluation of physical performance. Scand J Rehabil Med. 1975;7(1):13-31.

29. Wolf SL, Thompson PA, Morris DM, et al. The EXCITE trial: attributes of the Wolf Motor Function Test in patients with subacute stroke. Neurorehabil Neural Repair. 2005;19(3):194-205.

30. Platz T, Pinkowski C, van Wijck F, Kim IH, di Bella P, Johnson G. Reliability and validity of arm function assessment with standardized guidelines for the Fugl-Meyer Test, Action Research Arm Test and Box and Block Test: a multicentre study. Clin Rehabil. 2005;19(4):404-411.

31. Morris DM, Uswatte G, Crago JE, Cook EW, Taub E. The reliability of the wolf motor function test for assessing upper extremity function after stroke. Arch Phys Med Rehabil. 2001;82(6):750-755.

32. Nijland R, van Wegen E, Verbunt J, van Wijk R, van Kordelaar J, Kwakkel G. A comparison of two validated tests for upper limb function after stroke: the Wolf Motor Function Test and the Action Research Arm Test. J Rehabil Med. 2010;42(7):694-696.

33. Uswatte G, Taub E, Morris D, Light K, Thompson PA. The Motor Activity Log-28: assessing daily use of the hemiparetic arm after stroke. Neurology. 2006;67(7):1189-1194.
34. Page SJ, Fulk GD, Boyne P. Clinically important differences for the upper-extremity Fugl-Meyer Scale in people with minimal to moderate impairment due to chronic stroke. Phys Ther. 2012;92(6):791-798.

35. Lin KC, Hsieh YW, Wu CY, Chen CL, Jang Y, Liu JS. Minimal detectable change and clinically important difference of the Wolf Motor Function Test in stroke patients. Neurorehabil Neural Repair. 2009; 23(5):429-434

36. Park SW, Wolf SL, Blanton S, Winstein C, Nichols-Larsen DS. The EXCITE Trial: predicting a clinically meaningful motor activity $\log$ outcome. Neurorehabil Neural Repair. 2008;22(5):486-493.

37. Wolf SL, Winstein CJ, Miller JP, et al. Effect of constraint-induced movement therapy on upper extremity function 3 to 9 months after stroke: the EXCITE randomized clinical trial. JAMA. 2006;296(17): 2095-2104.

38. Tiozzo E, Youbi M, Dave K, et al. Aerobic, resistance, and cognitive exercise training poststroke. Stroke. 2015;46(7):2012-2016.

39. Compagnat M, Salle JY, Mandigout S, Lacroix J, Vuillerme N, Daviet JC. Rating of perceived exertion with Borg scale in stroke over two common activities of the daily living. Top Stroke Rehabil. 2018;25(2): $145-149$.
Clinical Interventions in Aging

\section{Publish your work in this journal}

Clinical Interventions in Aging is an international, peer-reviewed journal focusing on evidence-based reports on the value or lack thereof of treatments intended to prevent or delay the onset of maladaptive correlates of aging in human beings. This journal is indexed on PubMed Central, MedLine,

\section{Dovepress}

CAS, Scopus and the Elsevier Bibliographic databases. The manuscript management system is completely online and includes a very quick and fair peer-review system, which is all easy to use. Visit http://www.dovepress. com/testimonials.php to read real quotes from published authors. 